\title{
Special Stains Used to Identify Fungal Infections
}

\author{
Manuela Gonzales and Benjamin Gonzalez
}

Histological staining is an essential technique used within research and the healthcare industry. The need for consistent high-level skill has led to the development of many programs that certify trained individuals as histology technicians. These programs provide the foundational knowledge that allow students to understand and perform embedding, cutting and staining techniques that are frequently used in a medical or research lab setting. This training covers many general staining techniques such as hematoxylin and eosin (H\&E) or Masson's trichrome, however there are many unique stains that are not commonly taught through these programs. This often leads the technician to feel inadequately prepared when asked to perform a technique that they have not been trained for. An example of two stains that are used to detect fungal infections are the Grocott Methenamine Silver-Nitrate (GMS) stain and the Periodic Acid-Schiff (PAS) stain. These stains are longer procedures that require the use of reagents and positive controls that are difficult to obtain for a demonstration and therefore rarely taught in most technologist programs.

Fungal infections are generally morphologically diverse and can appear in tissues as hyphae, spores, or budding yeasts. Conventional stains such as H\&E can identify some classic features of fungi but cannot identify the species involved and is not usually recommended to diagnose fungal infections. This is because not all species are naturally pigmented, or they could be present in low numbers and overlooked using general staining.

The GMS stain is an oxidizing stain that uses chromic acid to form aldehydes from the polysaccharide rich cell wall of fungal pathogens (Grocott, 1955). When the tissue is treated with methenamine silver nitrate, the aldehyde groups will react with the silver nitrate generating a metallic silver which will appear black. The sample can be counter stained with Light green stain which provides greater contrast of the fungal structures. Some common errors associated with the GMS stain come from the tools used. Chromic acid staining requires that metallic tools and hardware be avoided. Other common issues can be attributed to insufficient removal of alcohols which can reduce the chromic acid, causing a visible change in the chromic acid solution (Carson, 2009).

The PAS stain is another common stain that uses the oxidation of fungal cell wall polysaccharides to visualize fungal infections using a periodic acid (Dadaci et al., 2015). This oxidation also results in the production of aldehydes that will react with a Schiff reagent causing a magenta color (Carson, 2009). The sample can be counterstained with hematoxylin. Glycogen can also be stained through the PAS reaction, which may be removed using a diastase digestion (Wang et al., 2017). A common issue encountered with PAS staining occurs when the Schiff reagent is overused. Schiff reagent can be tested by the dropwise addition to a $40 \%$ solution of formaldehyde. An immediate color change should occur. If there is a delay, then the reagent should be replaced.

These stains along with good embedding and sectioning techniques can be used to demonstrate the presence of a fungal infection. Positive controls are required when using either of these techniques which can be obtained from tissue samples infected with a known fungal pathogen. Although chromic acid is toxic and produces an increased risk for the technician, the GMS stain is less prone to nonspecific staining then the PAS stain (D'Hue et al., 2008; Carson, 2009). 


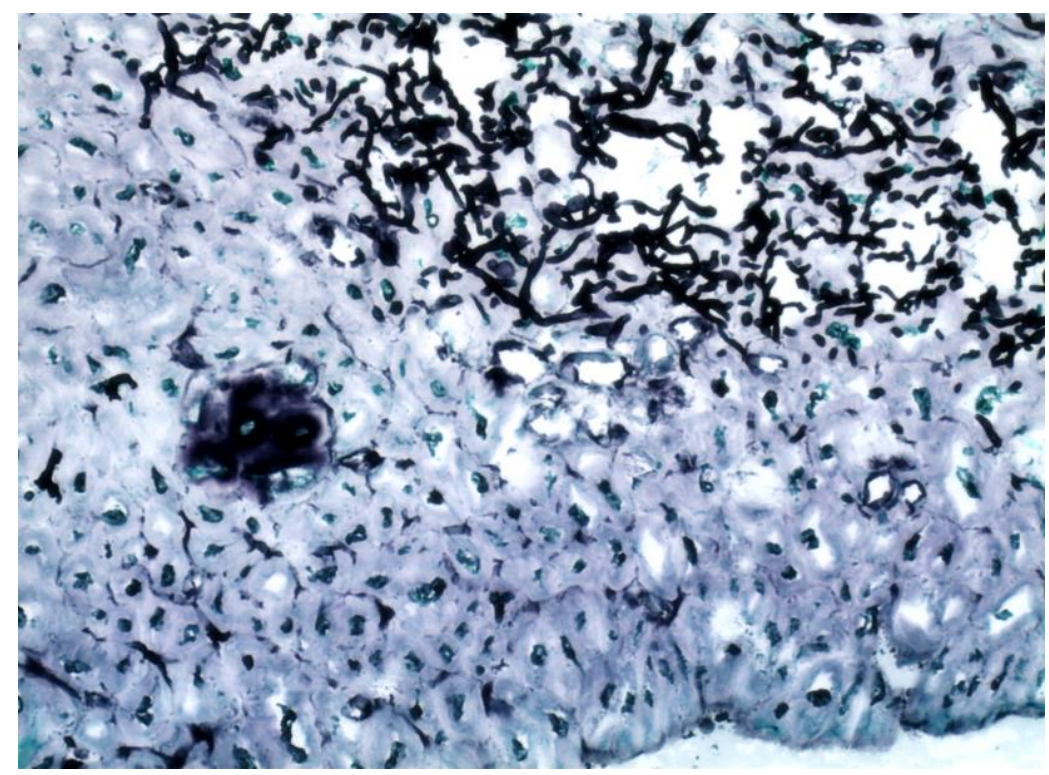

Figure 1. A GMS stain used as a positive control for diagnosis. The dark tubular structures are the stained fungal cell wall. The counterstain is light green.

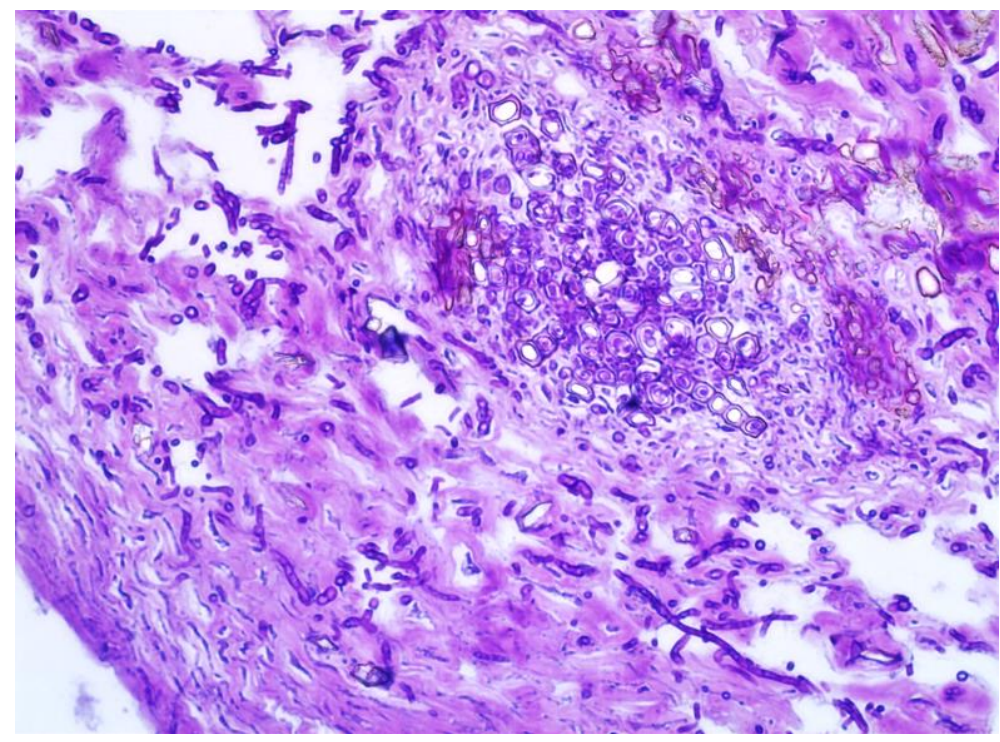

Figure 2. An example of a PAS positive control. The tubular magenta structures are the stained fungal cell wall. The counterstain is hematoxylin.

\section{References}

Grocott, R.G. 1955. A Stain for Fungi in Tissue Sections and Smears. American Journal of Clinical Pathology. 25:975-979. doi:10.1093/ajcp/25.8_ts.0975.

Carson, F.L., and C.H. Cappellano. 2009. Histotechnology. ASCP Press, Chicago.

Dadaci, Z., F. Kılınç, T.T. Ozer, G.O. Sahin, N.O. Acir, and M. Borazan. 2015. Periodic acid-Schiff staining demonstrates fungi in chronic anterior blepharitis. Eye. 29:1522-1527. doi:10.1038/eye.2015.144.

DHue, Z., S.M. Perkins, and S.D. Billings. 2008. GMS is superior to PAS for diagnosis of onychomycosis. Journal of Cutaneous Pathology. 35:745-747. doi:10.1111/j.1600-0560.2007.00890.x. 